\title{
The Inductive Inverse Kinematics Algorithm to Manipulate the Posture of an Articulated Body
}

\author{
Jin Ok Kim ${ }^{1}$, Bum Ro Lee ${ }^{2}$, Chin Hyun Chung ${ }^{2}$, Jun Hwang ${ }^{3}$, and \\ Woongjae Lee $^{3}$
}

1 School of Information and Communication Engineering, Sungkyunkwan University, 300, Chunchun-dong, Jangan-gu, Suwon, Kyunggi-do, 440-746, KOREA

jinny@ece.skku.ac.kr

2 Department of Information and Control Engineering, Kwangwoon University,

447-1, Wolgye-dong, Nowon-gu, Seoul, 139-701, KOREA

chung@kw.ac.kr

3 Division of Information and Communication Engineering, Seoul Women's University, 126, Kongnung2-dong, Nowon-gu, Seoul, 139-774, KOREA

wjlee@swu.ac.kr

\begin{abstract}
Inverse kinematics is a very useful method for controlling the posture of an articulated body. In most inverse kinematics processes, the major matter of concern is not the posture of an articulated body itself, but the position and direction of the end effector. In some applications such as $3 \mathrm{D}$ character animation, however, it is more important to generate an overall natural posture for the character rather than to place the end effector in the exact position. Indeed, when an animator wants to modify the posture of a human-like 3D character with many physical constraints, he has to undergo considerable trial-and-error to generate a realistic posture for the character. In this paper, the Inductive Inverse Kinematics (IIK) algorithm using a Uniform Posture Map (UPM) is proposed to control the posture of a human-like 3D character. The proposed algorithm quantizes human behaviors without distortion to generate a UPM, and then generates a natural posture by searching the UPM. If necessary, the resulting posture could be compensated with a traditional Cyclic Coordinate Descent (CCD). The proposed method could be applied to produce 3D-character animation based on the key frame method, 3D games and virtual reality.
\end{abstract}

\section{Introduction}

In traditional animation production, huge amounts of time are spent drawing each frame manually. With the aid of computer graphics, the tedious processes have been automated to make the overall production process more efficient. Key frame animation is a very useful method for producing successive frames automatically in character animation. If an animator sets several key frames, the computer system automatically generates intermediate frames among the key frames. Therefore, it is very important for an animator to set the key frames 
properly [1] [2] [3]. Many animators use the inverse kinematics algorithm to control the posture of a 3D character [4] [5]. Inverse kinematics technique adopted from robotics has the potential to relieve the animator from deciding the specification of every motion parameter within a frame. It calculates the angles of each joint of the limbs when the end effector points to a specific place. Thus, an animator can control the posture of the limbs by dragging the end effector. In character animation that uses motion capture, the inverse kinematics algorithms are also available to correct the motion capture error by improving false posture that does not observe physical laws. If an animator wants to control the $3 \mathrm{D}$ character with many physical constraints, he actually has to undergo considerable trial-and-error to generate a realistic posture. The Cyclic Coordinate Descent (CCD) algorithm [6] [7] is a heuristic inverse kinematics algorithm, that is suitable for interactive control of an articulated body. Like other inverse kinematics algorithms, it can generate many different resulting postures in accordance with the initial posture. However, it is very difficult to choose a feasible posture among many resulting postures. To reform these problems, the Inductive Inverse Kinematics (IIK) algorithm using a Uniform Posture Map (UPM) is proposed in this paper to control the posture of human-like characters. The UPM is organized through the quantization of various postures with an unsupervised learning algorithm, and the learning algorithm prevents the generating of invalid output neurons. This guarantees that the postures generated by the UPM are realistic postures which observe physical constraints. Therefore, it is possible to get a natural posture by finding a posture whose forward kinematics point is closest to its desired position. Because most animators are concerned about the desired natural posture itself rather than the exact position and direction of end effector, the IIK using UPM contributes to the production of character animation efficiently. The IIK algorithm consists of three phases. First, we generate a UPM that reflects the motion tendency of an articulated body and find a proper inverse kinematics solution in generating the UPM. Then, if necessary, we compensate the solution by using the CCD algorithm. This method can be applied to produce 3D-character animation based on the key frame method, 3D games and virtual reality.

\section{Inductive Inverse Kinematics}

\subsection{Inverse Kinematics Algorithm}

Most industrial robotic systems have an end effector at the end of the kinematics chains. To control the position and direction of the end effector, the controller has to calculate the proper angles of each joint. To calculate the proper angle set, the inverse kinematics has been researched according to various points of view. As a natural consequence, the major matter of concern is not the posture itself of an articulated body, but the position and direction of the end effector in most inverse kinematics processes. Indeed, inverse kinematics algorithms using the Jacobian matrix [8] and energy constraints [9] focus on calculating 
the position of the end effector [10] [11] [12]. In some applications, such as 3Dcharacter animation, however, it could be more important to generate a natural and realistic posture rather than the exact position and direction of the end effector [13] [14] [15]. Because most 3D animation producing tools adopt the traditional inverse kinematics algorithm, when an animator wants to control the $3 \mathrm{D}$ character with physical constraints using tools, he actually has to undergo considerable trial-and-error to generate a realistic posture. For these reasons, it is necessary to develop the special inverse kinematics algorithm to control the posture of human-like configurations. For these practical needs, the inductive inverse kinematics algorithm using a Uniform Posture Map (UPM) is proposed, and compared with a traditional inverse kinematics algorithm of a Cyclic Coordinate Descent (CCD).

\subsection{Cyclic Coordinate Descent Algorithm}

The Cyclic Coordinate Descent algorithm is an iterative heuristic search technique to minimize position and orientation errors by varying each joint variable one at a time. In each iteration, its joint variable is modified in turn to minimize an error function. The calculation at each joint is so cheap that we found its solution very quickly. In the progress of minimizing the error function, the position and orientation of the end-effector are conversed to the desired value. Algorithm 1 shows the CCD Algorithm:

Although the CCD algorithm performs satisfactorily in an interactive posture control, its result is dependent on its initial posture. In other words, it results in many different solutions in accordance with its initial conditions. Among the many solutions, some solutions are suitable for application to the humanlike character animation but others are not. Thus the animator has to conduct several experiments to get a satisfactory result.

\subsection{Inductive Inverse Kinematics Algorithm}

It is difficult to control the posture of a human-like articulated body using physical constraints through general inverse kinematics, because its major objective is to locate the end effector at the desired point with the desired direction. Except for the case of obstacle avoidance, most inverse kinematics algorithms have been developed from this point of view. If the control target is not an industrial robotic system but the arm or leg of a human-like 3D character, the natural posture itself could be the major purpose of the inverse kinematics. In such applications, many animators have used the traditional inverse kinematics to generate a natural posture through trial-and-error or multiple applications to a partial joint. Therefore it is necessary to research the inverse kinematics algorithm from a different viewpoint than that of the robot control. In this paper, we propose the Inductive Inverse Kinematics (IIK) algorithm using a UPM. This algorithm consists of two phases and one optional phase. First we generated a uniform posture map which reflected the motion tendency of a human-like articulated body. In this phase, a pre-calculated forward kinematics table is recommended. 


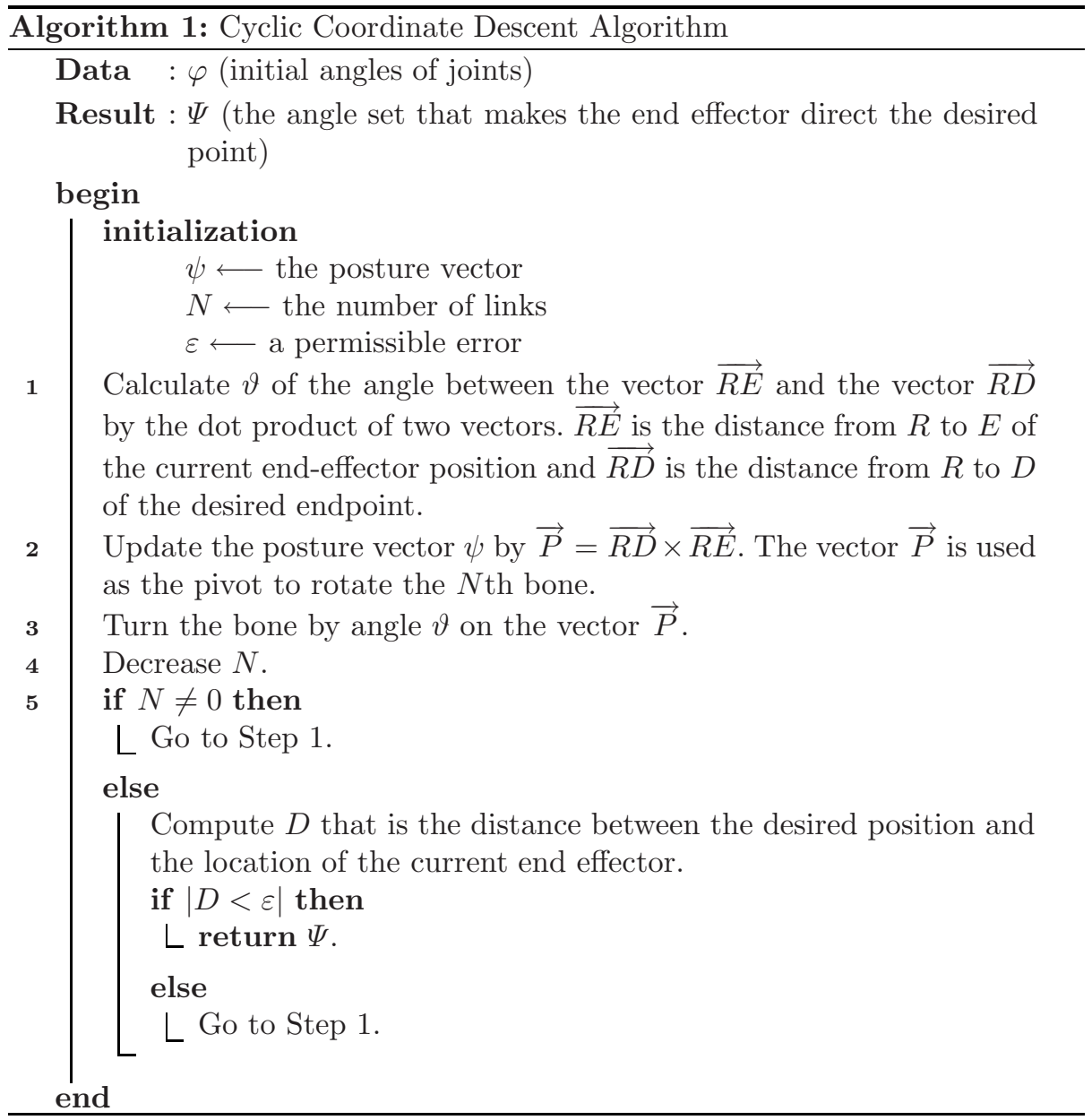

The forward kinematics table contains the forward kinematics values of each output neuron and it contributes to the reduction of the computational cost in a run-time. In the second phase, we searched the forward kinematics table to find the point with the smallest distance from the desired point, and to choose the posture vector $Q$ associated with the point. In most cases, the posture vector $Q$ has a realistic nature, and its end effector is close enough to the desired point. If an animator wants to make the current end point approach desired point more closely, he could use the traditional CCD algorithm in an optional phase. In the optional phase, we compensated for the solution using the CCD algorithm with constraints according to the DOF range table. The DOF table contains the ranges of each DOF acquired from an experiment and it constrains the degree of rotation of joint through the traditional CCD algorithm. In each step, we checked the calculated rotation angle $\vartheta$ whether the resulting angle beyond the limit specified in DOF range table. If the rotation angle $\vartheta$ made the DOF exceed 
its DOF range, we substituted $\vartheta$ with $\vartheta / 2$, and checked again. This step was repeated until we could find $\vartheta$ observing the constraint, or the number of repetition reaching the repetitional limit. If we could find a satisfactory $\vartheta$, we rotated the joint by $\vartheta$; otherwise we processed the next joint. We repeated such entire iteration until the end effector is close enough to the desired position. Algorithm 2 describes the inductive inverse kinematics algorithm.

If the proposed algorithm is applied to control a human-like 3D character, it is possible to get the available posture vector. In robot application, it could be important to get an exact position and direction of the end effector in a 3D space. On the other hand, 3D-character animation production does not require calculations for an exact position and directions of the end effector, but the control of the posture of the character following common behavior patterns. Therefore it is not absolutely necessary to compensate for the resulting posture with the optional posture-compensation process.

\section{Experiment and Result}

The inductive inverse kinematics algorithm consists of three separate processes: the UPM leaning process, the posture extraction using the UPM, and the optional compensation for the posture using the traditional CCD algorithm. In the UPM leaning process, we used the actual human motion capture data which was acquired with the optical motion-capture system. The right leg of the applied human character was chosen as a testing limb out of the entire skeletal hierarchy, consisting of 3 joints. Then we set a map topology with 9 input neurons, trained the posture map and generated 1,608 output neurons. After generating a UPM, we calculated the distance between the desired position and all positions of the end effector associated with each output neuron, and selected an output neuron with a minimum distance. If the minimum distance exceeded the predefined acceptable range, we compensate for the resulting posture using the traditional CCD.

Figure 1 shows the variation of the right leg, while the desired point is moved from the point $(20,-60,-70)$ to the point $(20,-45,-50)$. The result proves that the right leg preserves its physical characteristics despite of the movement of the end effector.

Figure 2 shows different results from the CCD and IIK. Figures 2(a) and 2 (b) have a desired point $(20,-40,10)$ and $2(\mathrm{c})$ and $2(\mathrm{~d})$ have a desired point $(65,-30,0)$, and $2(\mathrm{e})$ and $2(\mathrm{f})$ have a desired point $(-4,-50,-65)$. Figures $2(\mathrm{a})$, $2(\mathrm{c})$ and 2(e) are results from the CCD algorithm and 2(b), 2(d) and 2(f) are results from the IIK algorithm. In Fig. 2, a small pink ball (upper ball) specifies the root of the right leg and the cyan ball (bottom ball) specifies the desired position. Three gray balls attached to the end of each links specify the joint. The third link from its root is the foot link. Figures $2(\mathrm{~b}), 2(\mathrm{~d})$ and $2(\mathrm{f})$ show that the IIK algorithm observes the physical constraints, and preserves the nature of posture. On the other hand, Figures 2(a), 2(c) and 2(e) show that the CCD algorithm does not consider the nature of posture. 
Algorithm 2: Inductive Inverse Kinematics Algorithm

Data : $\varphi$ (initial angles of joints)

Result : $\Psi$ (the angle set that makes the end effector direct the desired point)

\section{begin}

\section{initialization}

$$
\begin{aligned}
& \psi \longleftarrow \text { the posture vector } \\
& N \longleftarrow \text { the number of links } \\
& C \longleftarrow \text { a watchdog counter } \\
& \varepsilon \longleftarrow \text { a permissible error } \\
& \eta \longleftarrow \text { a permissible count }
\end{aligned}
$$

Generate a uniform posture map and build a DOF range table and a forward kinematics table.

Search the forward kinematics table to find the point with smallest distance from the desired point.

Calculate $\vartheta$ of the angle between the vector $\overrightarrow{R E}$ and the vector $\overrightarrow{R D}$ by the dot product of two vectors. $\overrightarrow{R E}$ is the distance from $R$ to $E$ of the current end-effector position and $\overrightarrow{R D}$ is the distance from $R$ to $D$ of the desired endpoint.

Calculate $\vec{P}=\overrightarrow{R D} \times \overrightarrow{R E}$. The vector $\vec{P}$ is used as the pivot to rotate $N$ th bone.

if the angle $\vartheta$ makes the DOF out of its DOF range then

$$
\begin{aligned}
& \vartheta=\vartheta / 2 . \\
& C=C+1 . \\
& \text { if } C<\eta \text { then } \\
& L \text { Go to Step } 5 . \\
& \text { else } \\
& L \text { Go to Step } 6 .
\end{aligned}
$$

L Rotate the bone by $\vartheta$ on the $\vec{P}$.

$$
\mathrm{L} \text { Go to Step } 2 .
$$

else

Compute $D$ that is the distance between the desired position and the location of the current end effector.

$$
\text { if }|D<\varepsilon| \text { then }
$$

$\llcorner\operatorname{return} \Psi$.

\section{else}

L Go to Step 2. 


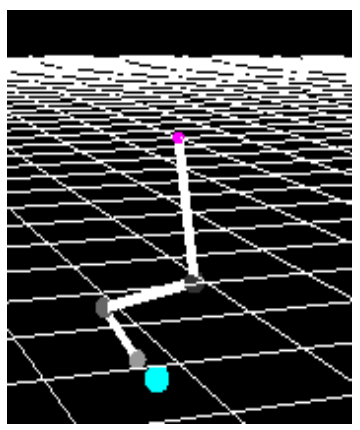

(a) $y=-60$

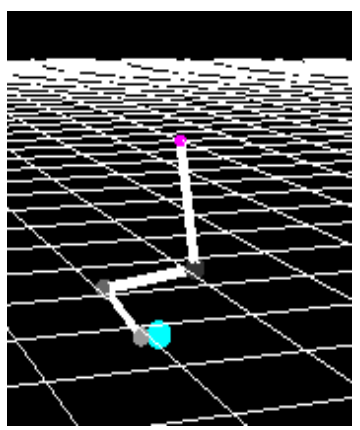

(c) $y=-50$

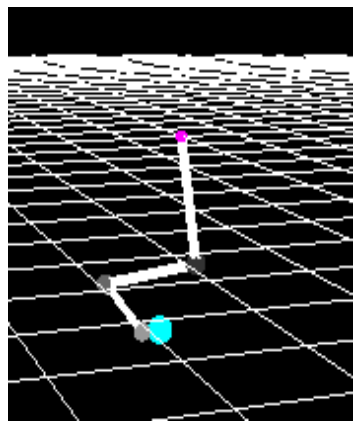

(b) $y=-55$

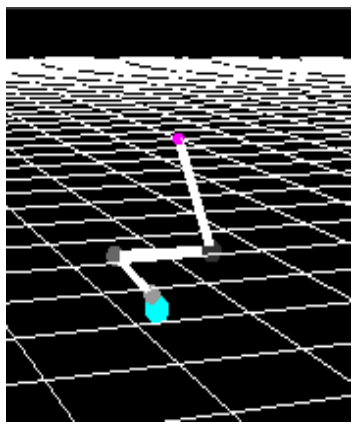

(d) $y=-45$

Fig. 1. Results of applying IIK according to variations of the axis $y$

\section{Conclusion}

The inverse kinematics method is used to control the posture of an articulated body in key frame animation production, but has some common problems in spite of its convenience. A common problem is that most inverse kinematics algorithms have multiple solutions for a desired point. If an articulated body has many physical constraints like the human body, the multiple solutions can be divided into two classes: some solutions represent existing posture; others represent unreal posture. With the traditional key-frame method, this problem is solved by repetitious trials and errors by applying inverse kinematics algorithms to each joint separately. However, this requires considerable cost and time. In this paper, the inductive inverse kinematics algorithm is proposed to solve this problem, and we prove that the inductive inverse kinematics can place the end effector on a desired position at a specific time, and that the resulting posture 


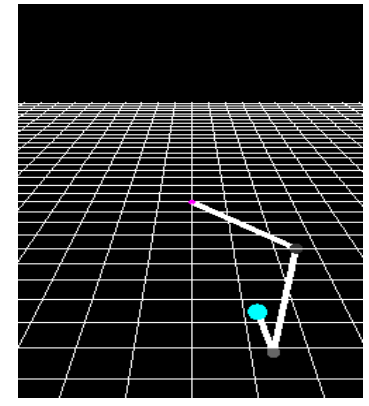

(a) Example 1 using CCD

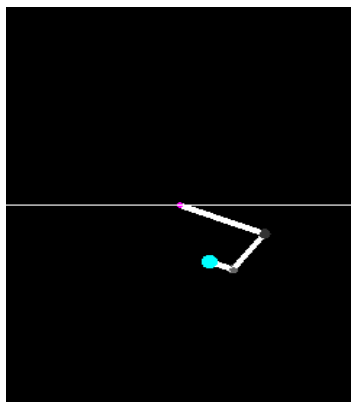

(c) Example 2 using CCD

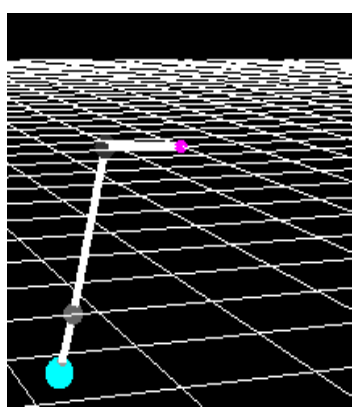

(e) Example 3 using CCD

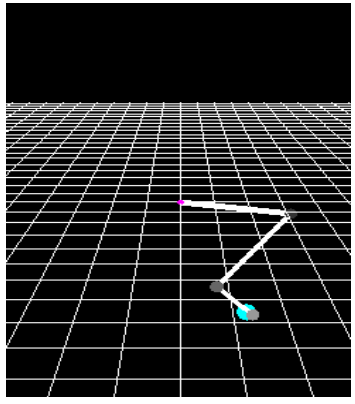

(b) Example 1 using IIK

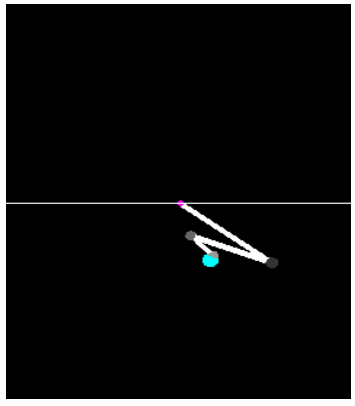

(d) Example 2 using IIK

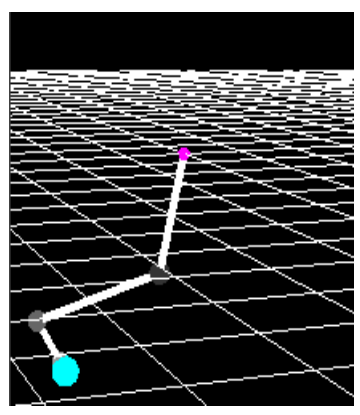

(f) Example 3 using IIK

Fig. 2. Comparison of result 
observes physical constraints. Inductive inverse kinematics algorithm increases efficiency to produce the desired key-frame in the animation production and reduces production costs and time. The inductive inverse kinematics algorithm can be applied to various fields such as 3D-character animation, 3D games, and virtual reality.

\section{References}

1. Brand, M., Hertzmann, A.: Style machine. In: Proc. of SIGGRAPH 2000, ACM Press (2000) 183-192

2. Grzeszczuk, R., Terzopoulos, D., Hinton, G.: Neuro animator: Fast neural emulation and control of physics-based models. In: Proc. of SIGGRAPH 98, ACM Press (1998) 9-20

3. Martinetz, T.M., Berkovich, S.G., Schulten, K.J.: Neural-gas network for vector quantization and its application to time-series prediction. IEEE Transactions on Neural Networks 4 (1993) 558-568

4. Shin, H.J., Lee, J., Shin, S.Y., Gleicher, M.: Computer puppetry. ACM Transactions on Graphics 20 (2001) 67-94

5. Popović, Z.: Controlling physics in realistic character animation. Communications of the ACM 43 (2000) 50-58

6. Welman, C.: Inverse Kinematics and Geometric Constrains for Articulated Figure Manipulation. Master dissertation, Simon Fraser University (1993) Dept. of Computer Science.

7. Watt, A., Watt, M.: Advanced Animation and Rendering Techniques: Theory and Practice. Addition Wesley, New York (1992)

8. Whitney, D.E.: Resolve motion rate control of manipulators and human prostheses. IEEE Transactions on Man-Machine Systems 10 (1969) 47-53

9. Badler, N.I., Zhao, J.: Real-time inverse kinematics with joint limit and spatial constraints. Technical Report TR-MS-CIS-89-09, University of Pennsylvania (1989)

10. Zhao, J., Badler, N.I.: Inverse kinematics positioning using nonlinear programming for highly articulated figures. ACM Transactions on Graphics 13 (1994) 313-336

11. Cohen, M.F.: Interactive spacetime control for animation. In: Proc. of SIGGRAPH 92, ACM Press (1992) 293-302

12. Wang, L.C.T., Chen, C.C.: A combined optimization method for solving the inverse kinematics problem of mechanical manipulators. IEEE Transactions on Robotics and Automation 7 (1991) 489-499

13. Fritzke, B.: Growing grid - a self-organizing network with constant neighborhood range and adaptation strength. Neural Processing Letters 2 (1995) 9-13

14. Kohonen, T., Huang, T.S., Schroeder, M.R.: Self-Organizing Maps. 3 edn. Springer Verlag (2000)

15. Baraldi, A., Alpaydin, E.: Constructive feedforward art clustering networks. IEEE Transactions on Neural Networks 13 (2002) 662-677 